\title{
Non-intubated, total intravenous anaesthesia proposed as a safe method for paediatric dentistry in a rural area
}

\author{
Tomasz Nikodemski ${ }^{1}$, Rafał Rojek² , Arkadiusz Kazimierczak ${ }^{3}$, Leszek Sagan ${ }^{4}$, Jeremy Clark ${ }^{5}$, \\ Katarzyna Ostrowska-Clark ${ }^{6}$, Zuzanna Nikodemska' \\ ${ }^{1}$ Anaesthetic Department, 'Dobra' Medical Centre, Dobra, Poland \\ ${ }^{2}$ Department of Paediatric Dentistry, Pomeranian Medical University, Szczecin, Poland \\ ${ }^{3}$ Clinic of Vascular Surgery, Pomeranian Medical University, Szczecin, Poland \\ ${ }^{4}$ Clinic of Neurosurgery and Children's Neurosurgery, Pomeranian Medical University, Poland \\ ${ }^{5}$ Department of Clinical and Molecular Biochemistry, Pomeranian Medical University, Szczecin, Poland \\ ${ }^{6}$ Surgery Department, Medimel, Szczecin, Poland
}

Nikodemski T, Rojek R, Kazimierczak A, Sagan L, Clark J, Ostrowska-Clark K, Nikodemska Z. Non-intubated, total intravenous anaesthesia proposed as a safe method for paediatric dentistry in a rural area. J Pre-Clin Clin Res. 2016; 10(1): 34-38. doi: 10.5604/18982395.1208186

\begin{abstract}
Introduction. General anaesthesia can reduce child stress associated with occasionally very unpleasant dental treatment. However, general anaesthesia with endotracheal intubation is commonly used with dental procedures despite the fact that endotracheal tubes obstruct good access to molars, especially in very small children. In this article we would like to contribute to changes in anaesthetic methods to those less harsh for patients.

Materials and method. At our dental practice, located at a rural area, total intravenous anaesthesia with propofol infusion, without endotracheal intubation, has been used for more than 10 years as standard procedure for the dental treatment of children. Retrospective analysis of medical records of 614 children was performed, including search for perioperative critical incidents.

Results. There were two adverse events. In the first, a boy (age 10.5 years with a history of tricuspid valve regurgitation) developed severe bradycardia, with no conjunction with any desaturation. Atropine was given and heart rhythm returned to normal values. The second incident occurred during the recovery of a 4-year-old girl who developed laryngospasm after the procedure, and the decision to use rescue intubation was made. The child recovered and was extubated shortly afterwards without any further problems. These two cases account for the total perioperative critical incident rate of $0.33 \%$ (95\% confidence interval $\sim 0$ to $1.3 \%$ ).

Conclusions. General anaesthesia without endotracheal intubation has been safe for paediatric dental treatment at our practice. Patients should be guided to follow strict fasting rules, and a throat pack and efficient suction are essential. The anaesthesiologist should be present during the intraoperative period until the child is safely discharged.
\end{abstract}

\section{Key words}

paediatric dentistry, general anaesthesia, airway management

\section{INTRODUCTION}

Dental treatment for children under general anaesthesia is a common practice in Europe. This treatment reduces child stress normally associated with sometimes very unpleasant dental procedures. If numerous large cavities are present, or with multiple extractions, neither local anaesthesia nor sedation provide adequate child comfort. If this results in lack of cooperation from the child then general anaesthesia is the most appropriate method of choice.

Historically, general anaesthesia using endotracheal intubation is the recommended option, despite the fact that it is well known that endotracheal intubation itself can cause difficulties. The endotracheal tube may partially obstruct the view of a dental surgeon, and even careful placement, proper fixation and small size often does not eliminate this problem. With many dental procedures the endotracheal tube obstructs good access to molars, especially in conjunction

Address for correspondence: Tomasz Nikodemski, Consultant Anaesthetist, 'Dobra', Medical Centre, Jasminowa 4, 72-003 Dobra, Poland

E-mail: nikodemski@me.com

Received: 06 June 2015; accepted: 15 June 2016 with poor mouth opening in very small children. In such situations numerous anaesthesiologists make the decision to use nasal intubation as the only appropriate approach. Nasal intubation is even more complicated, requires higher anaesthetic skills and has a higher critical incident rate, but despite this, it is still the most frequently used method. This raises the following questions: Do we really need to make anaesthesia very complex? Are more sophisticated and complicated methods necessarily safer?

In modern anaesthetic practice there are various changes being made towards methods which are less harsh for the patient, and some other examples are given here. A new perioperative fasting guideline has recently been published which has challenged several dogmas previously believed in for many years $[1,2]$. For example, today we do not fast patients for unnecessarily long periods of time. In modern intensive therapy, non-invasive ventilation is gradually becoming a more frequentlyused approach. Similarly, the Enhanced Recovery After Surgery (ERAS) protocols have encouraged us to change daily practice in many fields and have tried to introduce new attitudes towards anaesthesia. In this article we would like to contribute to these changes in attitudes by 
suggesting a method for anaesthesia which is less harsh than those currently promoted, and it is possible that sharing our experience may be useful for other anaesthesiologists in everyday practice.

Our dental practice was established in 2003 in a small village, and at the beginning endotracheal intubation was not used as a standard procedure for dental treatment for children, but rather general anaesthesia without endotracheal intubation. This procedure has been used occasionally before (e.g. Wang et al.) [3] but few data are available concerning outcome. Endotracheal intubation was performed only with extended extractions, classified by the surgeon as 'difficult' or when any emergency condition arose.

\section{OBJECTIVE}

The aim of this study was to determine whether performing general anaesthesia without endotracheal intubation is a relatively safe method, and can therefore lead to improvements in surgical access. Possible dangers from the described protocol are also discussed, but these are not thought to out-weigh the benefits described.

\section{MATERIALS AND METHOD}

Approval of the Ethical Committee at the Regional Medical Chamber in Szczecin, Poland, was obtained (Ref. No. 16/KB/ IV/2012, April 2012). The study was performed in accordance with the Helsinki Declaration, Version 17c.

After approval from the Ethical Committee, a retrospective analysis of medical records was performed. Between March 2003 - October 2012, 658 patients were anaesthetised at our practice (among whom were 44 adults, excluded from the present study). Data from child patients $(n=614)$ with maximum age $<16$ years (y) (mean $4.7 \mathrm{y}$; minimum $1.0 \mathrm{y}$, maximum 16.0 y, s.d. 2.5 y) were used for statistical analysis. In this group there were $336(54.7 \%)$ boys and 278 (45.3\%) girls (Fig. 1).

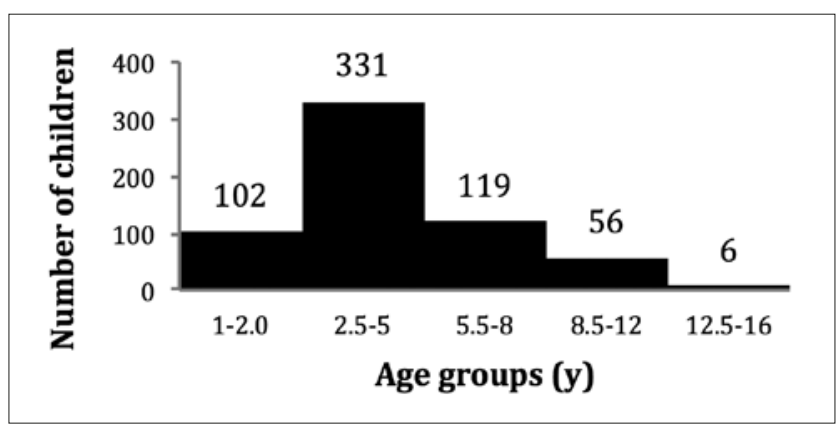

Figure 1. Age distribution of paediatric patients who underwent dental surgery

A search of medical records for previous medical problems was performed for each child. Demographic data (age, gender, mass) were evaluated, as well as past medical history (presence of comorbid conditions such as valvular heart disease, hypertension, coronary artery disease, asthma, COPD, epilepsy, cerebral palsy, autism, or diabetes).

Anaesthetic technique was assessed, taking into account the type and total dose of medication used, as well as haemoglobin oxygen saturation values during anaesthesia and recovery, duration of anaesthetic administration, and method of securing the airway (laryngeal mask airway or endotracheal tube). Inhalation induction was usually initiated via a face mask with sevoflurane, up to $6 \%$ by volume, in oxygen with a fresh gas flow of 6 litre min $^{-1}$ Typical anaesthesia included total intravenous anaesthesia with propofol, fentanyl, midazolam and paracetamol. The propofol infusion rate was started according to our protocol $(13,11$, 10, 9, and then $7 \mathrm{mg} / \mathrm{kg} / \mathrm{h}$ each for approx. 10-15 min.) and adjusted individually to achieve a lack of patient's response to surgical stimuli.

The range of dental treatment was assessed. Patients had had either dental fillings and root canal treatments ('conservative treatment') or conservative treatments plus tooth extraction ('non-conservative treatment'). The number and type of critical (or non-critical) incidents that had occurred during anaesthesia or recovery were evaluated. Confidence intervals were calculated using a modified Wald method (http://graphpad.com/quickcalcs/confInterval2). Assessed variables included:

- frequency of intubation requested by a surgeon;

- frequency of emergency intubation;

- rate of decline in oxygen saturation of haemoglobin in arterial blood $\left(\mathrm{SaO}_{2}\right)$ below $96 \%$ during general anaesthesia, and recovery time;

- frequency of perioperative critical incidents, defined as 'an event which led to harm or could have led to harm if it had been allowed to progress', according to a definition from The Royal College of Anaesthetists ${ }^{4}$

Also analysed were the frequency and type of critical incidents in comparative studies found in the literature. The databases Medline (US National Library of Medicine, Bethesda, USA), Google Scholar (Google Inc., Mountain View, California, USA) and http://www.knowledge.scot.nhs. uk were searched until March 2013. Keywords and/or mesh terms used were: 'Dental', 'General Anaesthesia', 'General Anesthesia', 'endotracheal tube', 'endotracheal intubation', 'perioperative critical incidents'.

The aim of our study was to assess whether tracheal intubation, during anaesthesia for dental treatment, can be safely omitted.

\section{RESULTS}

The average body mass in the paediatric group was $18.8 \mathrm{~kg}$ (range 8.5 - $85.0 \mathrm{~kg}$, s.d. 8.1). Most paediatric patients (581; 94.6\%)were found to be in ASA class 1 and few in class 2 (33; 5.4\%). There were no patients in ASA class 3 or 4 . Comorbidities are presented in Table 1.

Conservative dental treatment was performed in 390 (63.5\%) patients, while in 224 patients additional teeth extractions were performed ('non-conservative'; $36.5 \%$ ). The average treatment time was $1.4 \mathrm{~h}(0.1-3.5 \mathrm{~h}$, s.d. 0.5 h) (treatment times are given in Figure 2, which also shows the treatment time groups into which patients were divided.

During preparation for treatment no premedication was given but 6 hours of preoperative fasting was required. Following recent ESA recommendations, children were encouraged to drink clear fluids up to 2 hours before the procedure [2]. The main method of induction of anaesthesia, used in $585(95.3 \%)$ cases, was gas induction with oxygen 
Table 1. Reported comorbidities in paediatric patients admitted for dental surgery $(n=109)$

\begin{tabular}{|c|c|}
\hline Class of comorbidity. & Specific type (No. of individuals affected). \\
\hline $\begin{array}{l}\text { Respiratory problems } \\
n=13\end{array}$ & $\begin{array}{l}\text { Asthma (11) } \\
\text { Laryngitis (2) }\end{array}$ \\
\hline $\begin{array}{l}\text { Metabolic disorders } \\
n=78\end{array}$ & $\begin{array}{l}\text { Hypothyroidism (1) } \\
\text { BMl > 25: total (78) } \\
\text { Overweight - BMI 25-30 (61) } \\
\text { Obesity - BMI > } 30(18)\end{array}$ \\
\hline $\begin{array}{l}\text { Diseases of the nervous system } \\
n=7\end{array}$ & $\begin{array}{l}\text { Epilepsy (5) } \\
\text { Mental retardation (3) } \\
\text { Hypoxic brain injury (1) }\end{array}$ \\
\hline $\begin{array}{l}\text { Cardiac problems } \\
n=14\end{array}$ & $\begin{array}{l}\text { Hypertension (2) } \\
\text { Heart defects - total (12) } \\
\text { Atrial septal defect (2) } \\
\text { Ventricular septal defect after correction (3) } \\
\text { Fallot syndrome after correction (1) } \\
\text { Patent ductusarteriosus (3) } \\
\text { Tricuspid regurgitation (2) } \\
\text { Bicuspid aortic valve (1) }\end{array}$ \\
\hline $\begin{array}{l}\text { Genetic diseases } \\
n=4\end{array}$ & $\begin{array}{l}\text { Down Syndrome (2) } \\
\text { Apertsyndrome (1) } \\
\text { Pier Robin syndrome (1) }\end{array}$ \\
\hline $\begin{array}{l}\text { Other anatomical defects which } \\
\text { potentially might have had an } \\
\text { impact on the maintenance of a } \\
\text { patent airway }\end{array}$ & Cleft palate after correction (1) \\
\hline
\end{tabular}

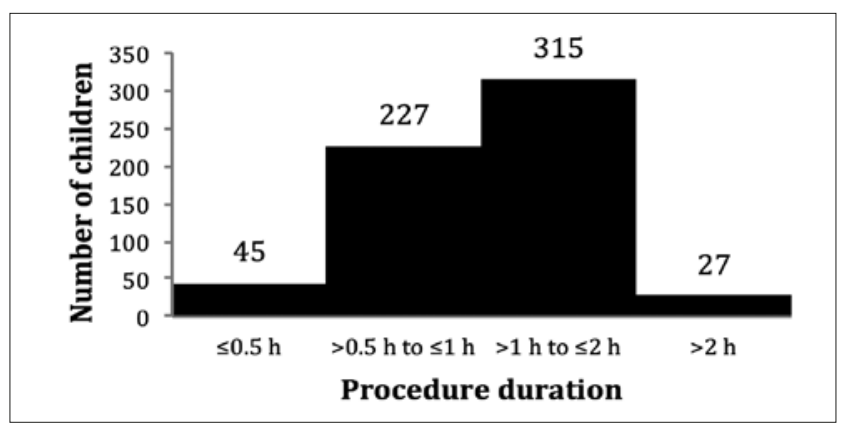

Figure 2. Distribution of paediatric dental patients according to treatment time

and sevofluran (see Method). For the remaining 29 (4.7\%) of children, intravenous induction with propofol was used.

After induction, an intravenous cannula was inserted and for 610 children propofol infusion started with a mean infusion rate of $8.7 \mathrm{mg} / \mathrm{kg} / \mathrm{h}$. The remainder, four children, did not receive propofol infusion because for these only one tooth extraction was performed using only an inhaled anaesthesia. The treatment time in these four cases was from 6-10 minutes.

During the operation, all patients patients were breathing spontaneously and oxygen was administered by nasal cannula (Plain Suction Catheter 8Fr). A second nasal cannula (Plain Suction Catheter 6Fr) was inserted into the opposite nostril to obtain sampling for capnography. During anaesthesia, standard monitoring, including ECG, $\mathrm{SaO}_{2}$, non-invasive blood pressure (NIBP), end-tidal carbon dioxide $\left(\mathrm{EtCO}_{2}\right)$ and anaesthesia gas monitoring were conducted.

There were 2 adverse events classified as critical incidents in our study population. In the first, a boy, age $10.5 \mathrm{y}$ with a history of tricuspid valve regurgitation, developed severe bradycardia after receiving conservative treatment (treatment time 1 hour).This incident had no conjunction with any desaturation. Atropine was given and heart rhythm returned to normal values. The second incident was observed during the recovery of a 4-year-old girl who had non-conservative treatment (treatment time $1.5 \mathrm{~h}$ ). In recovery, she developed laryngospasm, and a decision to use rescue intubation was made. The child recovered and was extubated shortly afterwards without any further problems. These 2 cases account for the total perioperative critical incident rate of $0.33 \%$ (95\% confidence interval $\sim 0$ to $1.3 \%$ ).

Four further cases were classified as non-critical incidents. In 2 cases (elective intubation) there was partial airway obstruction which caused problems in maintaining the airway before dental treatment was started and therefore, to preventan an adverse event, a laryngeal mask airway (LMA) was inserted. Subsequently, at the request of the surgeon, 2 patients were intubated on induction because of the extent of the surgery.

Advanced airway devices were therefore used in a total number of 5 cases $(0.81 \%)-2$ elective intubations, 2 LMA insertions during dental treatment (i.e. 4 non-critical incidents) and 1 emergency intubation (critical incident) in recovery.

For all patients, a pack (a moist cotton swab, $5 \times 5 \mathrm{~cm}$ ) was inserted into the oral cavity, and the dentist checked its position throughout the dental treatment. In all cases, rinsing water was used gently, as was attentive suction to prevent flooding of the mouth. In addition to the propofol during surgery some patients were also given Midazolam and Fentanyl or Midazolam and Ketamine. To treat postoperative pain a single dose of intravenous Morphine, Paracetamol or rectal Diclofenac was used. Local anaesthetic infiltration (Mepivacaine 3\%) was used for surgical procedures for all patients. Mean total doses of anaesthetics used during the proceduresare presented in Table 2. Mean time from end of anaesthesia to discharge home was between 35-60 min.

Table 2. Medication doses during anaesthesiafor paediatric patients $(n=614)$ admitted for dental surgery

\begin{tabular}{|c|c|c|c|c|c|c|}
\hline \multirow[t]{2}{*}{ Medication } & & \multirow{2}{*}{$\begin{array}{c}\text { No. of } \\
\text { patients } \\
\text { treated (\%) } \\
\text { Mean }\end{array}$} & \multicolumn{4}{|c|}{ Dose for those treated. } \\
\hline & & & $\begin{array}{l}\text { Standard } \\
\text { deviation }\end{array}$ & Minimum & Maximum & \\
\hline Infusion & $\begin{array}{l}\text { Propofol } \\
(\mathrm{mg} / \mathrm{kg} / \mathrm{h})\end{array}$ & 612 (99.7\%) & 8.7 & 0.32 & 21 & 3.4 \\
\hline \multirow{3}{*}{$\begin{array}{l}\text { Intra- } \\
\text { operative } \\
\text { medi- } \\
\text { cation }\end{array}$} & $\begin{array}{l}\text { Fentanyl } \\
\text { (mcg/kg) }\end{array}$ & $365(60 \%)$ & 1.1 & 0.57 & 0.29 & 4.4 \\
\hline & $\begin{array}{l}\text { Midazolam } \\
(\mathrm{mg} / \mathrm{kg})\end{array}$ & $555(90 \%)$ & 0.065 & 0.029 & 0.01 & 0.3 \\
\hline & $\begin{array}{l}\text { Ketamine } \\
(\mathrm{mg} / \mathrm{kg})\end{array}$ & $149(24 \%)$ & 1.4 & 1.6 & 0.26 & 20 \\
\hline \multirow{3}{*}{$\begin{array}{l}\text { Post- } \\
\text { operative } \\
\text { analgesia }\end{array}$} & $\begin{array}{l}\text { Morphine } \\
(\mathrm{mg} / \mathrm{kg})\end{array}$ & $5(0.8 \%)$ & 0.077 & 0.036 & 0.02 & 0.13 \\
\hline & $\begin{array}{l}\text { Paracetamol } \\
(\mathrm{mg} / \mathrm{kg})\end{array}$ & $71(12 \%)$ & 20 & 6.1 & 9.3 & 35 \\
\hline & $\begin{array}{l}\text { Diclofenac } \\
(\mathrm{mg} / \mathrm{kg})\end{array}$ & $33(5.4 \%)$ & 1.2 & 0.1 & 1.0 & 1.3 \\
\hline
\end{tabular}

No desaturation episodes (no fall in $\mathrm{SaO}_{2}$ below $96 \%$ for any period of time) were recorded on anaesthetic charts. $\mathrm{EtCO}_{2}$ was found to be within normal range during the whole procedure in all cases. Opioid and non-opioid analgesics were given according to the anaesthesiologist's preference (there was no standard acute pain protocol in our practice at that time). 


\section{DISCUSSION}

In many reports, the rate of failed sedation for diagnostic and therapeutic paediatric procedures is given in the range of $0.2 \%-50 \%[4,5,6,7]$. For example, for intramuscular ketamine injections for endoscopy, an observational study [5] $(n=60)$ gave inadequate sedation for infants: $50 \% ; 1-$ 7 -year-olds: $32 \%$; and over 7-year-olds: $6.7 \%$. Malviya et al. [6] reported inadequate sedation of $16 \%$ and failed sedation of 7\%, before MRI and CT scans, and Pane et al. [7] reported $2.3 \%$ adverse events following procedural sedation in an emergency department. In the presented study, the total perioperative critical incident rate was only $0.3 \%$, and therefore the methods used are promoted.

It is very difficult to determine the boundary between deep sedation and general anaesthesia, and here the general anaesthetic techniques used could well be regarded as a form of deep sedation. In our practice we used total intravenous anaesthesia, and propofol infusion was adjusted individually to achieve a lack of patient's response to surgical stimuli. This approach allowed us reduction of the likelihood of insufficient sedation and, consequently, failure of therapy.

However, during general anaesthesia for dental treatment the dentist and anaesthesiologist jointly need to access the patient's mouth. The introduction of the endotracheal tube through the mouth in order to secure a patent airway during anaesthesia makes access very difficult for the dentist, especially in cases involving young children. In these cases, many dentists will ask the anaesthesiologist for nasal intubation, which is fairly traumatic and introduces additional likely critical incidents.

A non-intubated anaesthetic technique was recently described by Wang et al. [3]. The authors paid particular attention to the possible difficulties that could occur during the procedure, but did not provide any data concerning how often various critical incidents occurred. We agree with Wang et al. that if non-intubated anaesthesia is chosen, then a very good partnership between the anaesthesiologist and the dentist is essential. The dentist must understand the importance of airway protection, usage of the throat pack and suction. Performing anaesthesia without intubation increases access to the mouth, thereby facilitating the work of the dentist.

Although improved access to the oral cavity can also be obtained by nasal intubation, it is, however, fraught with more critical incidents than oral intubation. The most common critical incidents from nasal intubation are epistaxis and nasal damage. According to El-Seify et al., pre-treating the nasal cavity with xylometazoline may reduce the frequency of epistaxis from $27.5 \%-7.5 \%$ [8]. Kim et al. recommended thermo-softening of the endotracheal tube to reduced the trauma related with nasal intubation, and this lowered the incidence of epistaxis from 52\%-22\% [9]. But despite these various manipulations and treatments, none of these publications demonstrated elimination of the consequences of nasal intubation [10].

Bleeding from the nose in the postoperative period is not only uncomfortable for the patient but, especially in young children, can cause airway obstruction which may lead to a life-threatening critical incident. In the data from our practice, no similar issue was recorded.

In 2012, Costaet al. analysed adverse events in children who had received a high dose of either chloral hydrate
(70-100 $\mathrm{mg} / \mathrm{kg}$ ) or midazolam (1 or $1.5 \mathrm{mg} / \mathrm{kg}$ ) during outpatient dental treatment [11]. The authors observed many adverse effects such as excessive sleep, irritation, dizziness or vomiting, among which excessive sleep was the most common and accounted for up to $24 \%$ of all cases treated with midazolam. In our practice, there were no similar adverse events. This allows us to hypothesize that total intravenous anaesthesia with propofol is much more predictable and controllable in an ambulatory setting for dental treatment than oral midazolam sedation.

In a large database of prospectively collected data $(30,037$ children from 26 institutions/practices) concerning paediatric sedation and/or anaesthesia for diagnostic and therapeutic procedures performed outside the operating room, there were no deaths according to a paper published by Cravero et al. [12]. In this group of patients, cardiopulmonary resuscitation was required once. Less serious events reported in that study were $\mathrm{O}_{2}$ desaturation below $90 \%$ for $>30$ seconds, occurring in approximately $1.57 \%$ ofsedations. Moreover, Cravero et al. reported that approximately $0.25 \%$ of procedures were associated with stridor, laryngospasm, wheezing or apnea, that could progress to a poor outcome if not managed well. Indeed, $0.5 \%$ sedations required airway and ventilation interventions ranging from bag-mask ventilation to oral airway placement or emergency intubation. In the same study group, vomiting (in a non-gastrointestinal procedure) occurred in approximately $0.5 \%$ of procedures.

In another study by Cravero et al., data from 49,836 propofol sedation/anaesthesia procedures were collected from 37 locations [13]. There were no deaths. Less serious events were more common with $\mathrm{O}_{2}$ desaturation below $90 \%$ for more than $30 \mathrm{~s}$, occurring in $1.54 \%$ of sedation/ anaesthesia administrations. Central apnea or airway obstruction occurred in $5.75 \%$ of sedation/anaesthesia administrations. In the same study, stridor, laryngospasm, excessive secretions, and vomiting had frequencies of $0.5 \%$, $0.96 \%, 3.41 \%$, and $0.49 \%$, respectively.

In the data from our practice there were no episodes of any desaturation below $96 \%$. We had one case of laryngospasm, which ended with a need for intubation for a short period of time with no further consequences. Most importantly, the total perioperative critical incident rate was only $0.33 \%$ (95\% confidence interval $\sim 0$ to $1.3 \%$ ).

However, in 2 further cases, when there was a problem to maintain the airway due to enlarged tonsils, the decision to insert reinforced LMA was made. Todd et al. compared endotracheal intubation and use of LMA for ambulatory oral surgery patients [14]. He concluded that LMA had an advantage over endotracheal intubation, the recovery time was quicker and anaesthetic costs were lower. We strongly agree with Todd et al. and would recommend using LMA in such situations.

Further possible theoretical dangers of the described protocol should be considered, for example, regurgitation, aspiration, or sudden obstruction by dental material. However, firstly, Ljungqvist et al. stated that the mean gastric fluid volume is in the range of $10-30 \mathrm{ml}$, with 120 $\mathrm{ml}$ rarely exceeded, irrespective of intake of clear fluids. During anaesthesia, passive regurgitation and pulmonary aspiration usually occurs only if the gastric content exceeds $200 \mathrm{ml}$ [1]. This explains why the risk of regurgitation when ESA perioperative fasting guidelines are complied is extremely small [2]. Secondly, vigilant airway management 
and avoidance of blowing air into the stomach will further reduce the risk of regurgitation and aspiration. Lastly, complications resulting from sudden obstruction by dental material are thought to be unlikely because the airway was protected by a purposely-placedand moistened swab.

The limitations of this study are its retrospective character, and the fact that only one practice was involved. Therefore, prospective studies from a larger number of practices are required to confirm these preliminary findings.

The issue of the cost of anaesthesia should also be raised, at the same time keeping in mind that an attempt to reduce this should not in any way increase the risk. Based on our experience, avoiding the routine use of advanced airwaydevices (LMA or endotracheal tube) does not increase the frequency of critical incidents due to anaesthesia and may lead to some savings.

\section{CONCLUSIONS}

General anaesthesia without endotracheal intubation has been safe for paediatric dental treatment at our rural practice. One requirement of this recommended method is close cooperation between the dentist, dental assistant, and anaesthesiologist. Patients should be guided to follow strict fasting rules to decrease the risk of gastric content aspiration, and a throat pack and efficient suction are essential. An experienced paediatric anaesthesiologist should be present during the intraoperative period until the child is safely discharged.

\section{Disclosure statement}

The authors received no financial suppport or sponsorship for their research and declare that they have no conflict of interests.

\section{REFERENCES}

1.Ljungqvist O, Søreide E. Preoperative fasting. Br J Surg. 2003; 90(4): 400-406.

2. Smith I, Kranke P, Murat I, Smith A, O’Sullivan G, Søreide E, et al. Perioperative fasting in adults and children: guidelines from the European Society of Anaesthesiology. Eur J Anaesthesiol. 2011; 28(8): 556-569.

3. Wang Y-C, Lin I-H, Huang C-H, Fan S-Z. Dental anesthesia for patients with special needs. Acta Anaesthesiol Taiwan. 2012; 50(3): 122-125.

4. Good Practice: A guide for departments of anaesthesia, critical care and pain management. 2006.http://www.rcoa.ac.uk/document-store/ the-good-practice-guide_(access: 2013.11.20)

5. Law AK, Ng DK, Chan K-K. Use of intramuscular ketamine for endoscopy sedation in children. Anaesthesia. 2003; 45(2): 180-185.

6. Malviya SS, Voepel-Lewis TT, Eldevik OPO, Rockwell DTD, Wong JHJ, Tait ARA. Sedation and general anaesthesia in children undergoing MRI and CT: adverse events and outcomes. Br J Anaesth. 2000; 84(6): 743-748.

7. Peña BM, Krauss B. Adverse events of procedural sedation and analgesia in a pediatric emergency department. Ann Emerg Med. 1999; 34(4 Pt 1): 483-491.

8.El-Seify ZA, Khattab AM, Shaaban AA, Metwalli OS, Hassan HE, Ajjoub LF. Xylometazoline pretreatment reduces nasotracheal intubation-related epistaxis in paediatric dental surgery. Br J Anaesth. 2010; 105(4): 501-505.

9. Kim YCY, Lee SHS, Noh GJG,Cho SY, Yeom JH, Shin WJ, et al. Thermosoftening treatment of the nasotracheal tube before intubation can reduce epistaxis and nasal damage. Anesth Analg. 2000; 91(3): $698-701$.

10. Mahajan R, Gupta R, Sharma A. Nasotracheal Intubation in Children. Anesthesiology. 2007; 107(5): 855-856.

11. Costa LR, Costa PS, Brasileiro SV, Bendo CB, Viegas CM, Paiva SM. Post-Discharge Adverse Events following Pediatric Sedation with High Doses of Oral Medication. J Pediatr. 2012; 160(5): 807-813.

12. Cravero JP, Blike GT, Beach M, Bendo CB, Viegas CM, Paiva SM, PhD5 et al. Incidence and Nature of Adverse Events During Pediatric Sedation/Anesthesia for Procedures Outside the Operating Room: Report From the Pediatric Sedation Research Consortium. Pediatrics. 2006; 118(3): 1087-1096.

13. Cravero JP, Beach ML, Blike GT, Gallagher SM, Hertzog JH. The incidence and nature of adverse events during pediatric sedation/ anesthesia with propofol for procedures outside the operating room: a report from the Pediatric Sedation Research Consortium. Anesth Analg. 2009; 108(3): 795-804.

14. Todd DW. A comparison of endotracheal intubation and use of the laryngeal mask airway for ambulatory oral surgery patients. The Lancet. 2002; 60(1): 2-4. 